\title{
Gene Editing: Do not forget about Animal Agriculture
}

\author{
Yanina Petracca ${ }^{3 *}$, Alison L. Van Eenennaam ${ }^{1}$ and Martin Alfredo Lema ${ }^{2}$ \\ ${ }^{1}$ Department of Animal Science, University of California, Davis, CA, USA \\ ${ }^{2}$ Chair of National Advisory Commission on Agricultural Biotechnology (CONABIA) and Professor at the National University of Quilmes, Argentina \\ ${ }^{3}$ Head of the GM Animal Biosafety Assessment Unit, National Ministry of Agro Industry, Argentina
}

Received: August 01, 2016; Accepted: August 07, 2016; Published: August 13, 2016

*Corresponding author: Yanina Lujan Petracca, Head of the GM Animal Biosafety Assessment Unit, National Ministry of Agro Industry, Argentina, E-mail: yaninapetracca@gmail.com

\section{To the Editor}

In vivo gene editing techniques have attracted great interest, not only in the scientific community, but also among regulatory agencies worldwide. Within the agricultural sector, gene-edited organisms pose an opportunity to help with broad challenges such as climate change, disease and pest pressure and increasing demands for agri food products [1]. Nevertheless, there are ongoing debates on how to handle such challenges at the regulatory level that need to be solved as a sine qua non condition for these organisms to have a chance of becoming commercially available.

Debates are more advanced in the case of biotech crops $[2,3,4]$. Where the term "New Breeding Techniques (NBT)"was coined to establish a differentiation with traditional transgenesis approaches that generate "Genetically Modified" Organisms (GMO). However, gene editing techniques are also being enthusiastically applied in the animal biotechnology field [5]. Moreover, in animals the "conventional" transgenic approach is inefficient and we anticipate most future products of animal biotechnology will be derived from gene editing techniques.

While traditional transgenesis led to the development of different crops found in the market today, animal biotechnology has not achieved an equivalent level of success. Technical difficulties and the uncertainty around regulatory frameworks disincentivize investment aimed at developing animal biotech products for food purposes. The complexity of processes such as cloning and DNA heterologous recombination hamper the generation of transgenic animals. Gene editing technologies, in contrast, have proven to be rapid and easy to apply in embryos and exhibit enhanced accuracy and efficiency rates [6,7]. Traits obtained include, inter allia, increases in yield and quality, as well as improvements in animal health and welfare $[8,9]$.

Most of these traits have been attained by small, precise changes in the genome and many do not introduce novel DNA sequences or may involve base pair deletions, reviving the debate on product-based versus process-based regulatory approaches. Taking into account those characteristics, Huang, et.al [3]. Argue that gene-edited crops that resemble those generated by conventional breeding or random mutagenesis should not be regulated. Most of what is stated in this commentary is compatible with the pioneering regulatory steps on NBTs recently published by Whelan, et.al [10]. For crops. However, these are essentially crop-oriented developments.

The increasing availability of innovative biotechnology tools for animal breeding makes it imperative to include gene edited animals alongside crops developed by NBT in the policy-making regulatory agenda. It is important that further steps towards an effective regulation for gene-edited or "NBT" agricultural products have a scope wide enough to include animal products simultaneously under the same general regulatory principles.

Additionally, given the nature of animal breeding, it will be portant to move away from an event-based to a trait-based regulatory paradigm. The introgression of approved event-based transgene into different germplasm that has been used in plantbreeding programs is not a viable approach for livestock breeding programs. Desired genetic edits will need to be accomplished in multiple founder animals in the nucleus breeding program, and regulatory frameworks need to be applicable to commercial animal breeding practices.

The developments in gene editing of live stock anticipate not only a wide range of opportunities but also new challenges to be faced in the near future. Having a clear regulatory pathway will provide an opportunity for these products, if shown to be effective and safe using appropriate science- and risk-based criteria, to contribute to the future of agricultural production systems and spare them the unfortunate fate of emblematic projects in applied animal transgenesis such as the Enviropig [11].

\section{Disclaimer}

Comments are those of the authors and don't prejudice the position of the institutions where they work.

\section{Competing Financial Interests}

The authors declare no competing financial interests. AVE acknowledges research support from the USDA for grants (201567015-23316; 2015-33522-24106) that involve genome editing in livestock. 


\section{References}

1. Petolino JF, Kumar S. Transgenic trait deployment using designed nucleases. Plant Biotechnol J. 2016;14(2):503-9. Doi: 10.1111/ pbi.12457.

2. Podevin N, Devos Y, Davies HV, Nielsen KM. Transgenic or not? No simple answer!. EMBO reports. 2012;13(12):1057-61. Doi: 10.1038/ embor.2012.168

3. Wolt JD, Wang K, Yang B. The Regulatory Status of Genome- edited Crops. Plant Biotechnol J. 2016;14(2):510-8. Doi: 10.1111/pbi.12444.

4. Huang S, Weigel D, Beachy RN, Li J. A proposed regulatory framework for genome-edited crops. Nat Genet. 2016;48(2):109-11. Doi: 10.1038/ng.3484.

5. Reardon S. Welcome to CRISPR zoo. Nature. 2016;531(7593):160-3 Doi: $10.1038 / 531160$ a.

6. Murray JD, Maga EA. Genetically engineered livestock for agriculture: a generation after the first transgenic animal research conference. Transgenic Res. 2016;25(3):321-7. Doi: 10.1007/s11248-016-99277.
7. Tan WS, Carlson DF, Walton MW, Fahrenkrug SC, Hackett PB. Precision Editing of Large Animal Genomes. Adv Genet. 2012;80:37-97. Doi: 10.1016/B978-0-12-404742-6.00002-8.

8. Crispo M, Mulet AP, Tesson L, Barrera N, Cuadro F, dos Santos-Neto $\mathrm{PC}$, et al. Efficient generation of Myostatin knock-out sheep using CRISPR/Cas9 technology and microinjection into zygotes. PLoS One. 2015;10(8):e0136690. Doi: 10.1371/journal.pone.0136690.

9. Wu H, Wang Y, Zhang Y, Yang M, Lv J, Liu J, et al. TALE nickasemediated SP110 knockin endows cattle with increased resistance to tuberculosis. Proc Natl Acad Sci USA. 2015;112(13):E1530-9. Doi: 10.1073/pnas.1421587112.

10. Whelan AI, Lema MA. Regulatory Framework for Gene Editing and other New Breeding Techniques (NBTs) in Argentina. GM Crops Food. 2015;6(4):253-65. Doi: 10.1080/21645698.2015.1114698.

11. Pollack A. Move to market gene-altered pigs in Canada is halted. New York Times, 2012; 3. 